\title{
Análisis de la incidencia en la aplicación de las normas reguladoras de la promoción y repitencia dentro del sistema educativo público costarricense en la gestión del Colegio Técnico Profesional de Limón
}

\author{
Analysis of the impact on the application of the rules governing the promotion and repetition within the \\ public education system in the management Rican Professional Technical College of Limon
}

\author{
Marlyn Jobson Vargas ${ }^{1}$ \\ Ministerio de Educación Pública \\ marjobson@costarricense.cr
}

Recibido 17 de octubre 2011 • Aceptado 10 diciembre 2012 • Corregido 19 de diciembre 2012

Resumen. La autora hace un breve análisis acerca de la incidencia en la aplicación de las Normas Reguladoras de la Promoción y Repitencia dentro del Sistema Educativo Público Costarricense en la gestión del Colegio Técnico Profesional de Limón. Se plantea la importancia de la administración educativa y su utilización como el medio por el cual se permite la ejecución de dichas normas y las estrategias que se han llevado a cabo en dicha institución para la implementación de las normas brindando acceso a aquellos estudiantes que han desertado del sistema educativo y que con estas normas de promoción y repitencia vigentes pueden retomarlo.

Palabras clave. Educación, administración de la educación, normas reguladoras, promoción, repitencia, calidad de la educación, deserción

Abstract. The author gives a brief analysis about the impact on the application of the rules governing the promotion and repetition within the public Costa Ricaneducation system in the management of the Professional Technical College of Limon. This raises the importance of educational administration and their use as the means by which it allows the implementation of these policies and strategies that have been implemented in the institution to apply the rules granting access to students who have dropped out of education system and promoting these standards in force and repetition can retake them.

Keywords. Education, education administration, regulatory standards, promotion, repetition, quality of education, dropout

\footnotetext{
Máster en Ciencias de la Educación con énfasis en Administración Educativa de la Universidad de Costa Rica (UCR). Bachiller y Licenciada en Ciencias de la Educación con énfasis en III Ciclo de la Educación General Básica y Educación Diversificada. Actualmente se desempeña como docente del Ministerio de Educación Pública en la provincia de Limón, Costa Rica.
} 


\section{Contextualización}

La ideología de la globalización, manifiesta entre los países, ha generado la búsqueda de la homogenización de conductas, patrones sociales, culturales, económicos y de otras áreas que han producido, a través de los años, reuniones, cumbres y otros eventos, en los cuales se analizan aspectos que promueven la implementación de los mismos objetivos de desarrollo; de tal manera que cada uno de los países participantes los ejecuten según su contexto y recursos disponibles.

En ese sentido, cada uno de los avances que ha logrado el ser humano como el fenómeno de la revolución tecnológica, ha incrementado el acceso a la información y al desarrollo de las personas y de las comunidades. Al respecto, Castells (2005) considera que son las tecnologías de la información quienes están modificando la sociedad, puesto que:

una revolución tecnológica, centrada en torno a las tecnologías de la información, está modificando la base material de la sociedad a un ritmo acelerado. Las economías de todo el mundo se han hecho interdependientes a escala global, introduciendo una nueva forma de relación entre economía, Estado y sociedad en un sistema de geometría variable. (p. 27)

De acuerdo con lo anterior, se puede apreciar como estos cambios promueven la reforma de los sistemas educativos que desarrolla cada país, de tal manera que no afecte su desarrollo económico, sino que, por el contrario, estos avances sean integrados dentro del proceso educativo, con el fin de promover el beneficio de éste y se sigan generando los recursos que las personas y la sociedad requieren para satisfacer sus necesidades y elevar su calidad de vida.

Derivado de las reformas que se han manifestado en los distintos países de América Latina, Costa Rica no es la excepción. La educación en Costa Rica, a través de los años, ha estado en constante cambio y crecimiento, lo que conlleva al mejoramiento de algunas de las deficiencias que poseía el sistema educativo y, a su vez, han surgido nuevas limitaciones que se deben reformar para lograr los objetivos propuestos en materia educativa. Por estas y otras razones, se han producido una serie de reformas que tienen como finalidad el mejoramiento del sector y el mejoramiento de la calidad de la educación costarricense.

\section{Justificación}

La preocupación manifiesta en el gobierno por el sector educativo, ha generado una serie de proyectos como ayudas económicas para la población estudiantil que no cuentan con los recursos 
económicos para costear los estudios; también, ayuda a la población que se encuentra en riesgo social, como el programa "Si es con drogas, no es conmigo", los cuales tienen como finalidad mejorar el servicio educativo que se le brinda a la sociedad, procurando llegar a todos los rincones del territorio nacional, cumpliendo de esta manera, con los objetivos trazados en la Ley Fundamental de Educación (1957) y en la Constitución Política de Costa Rica (1949).

Uno de los programas instaurados por el gobierno en el período 2006-2010, es el llamado "Avancemos", que brinda ayuda económica considerable para la población estudiantil que posee dificultades económicas. Asimismo, otro de los programas desarrollados, que a su vez reformuló algunos artículos del Reglamento de evaluación de los aprendizajes, del Ministerio de Educación Pública (MEP), fue el relacionado con las Normas de promoción y repitencia dentro del sistema educativo público costarricense.

La reforma a este reglamento pretende lograr la motivación de la población estudiantil por el estudio para que puedan continuar con el proceso educativo; de tal forma que se mantengan en las aulas hasta lograr obtener el título de bachilleres en la Educación Diversificada.

La implementación de esta normativa, luego de su publicación en el diario La Gaceta de Costa Rica, en el mes de noviembre, ya finalizando el curso lectivo del 2008 y vigente en la actualidad, ha suscitado varios acontecimientos o inconvenientes de carácter administrativo, de los cuales solo se tienen ciertas críticas y argumentos; por ejemplo, la dificultad en la confección de los horarios en las instituciones de educación secundaria, el aumento de estudiantes que poseen tiempo de ocio y no lo invierten de la mejor manera, provocando un caos en los pasillos de los centros educativos; además, el abarrotamiento de algunas secciones debido a que la población estudiantil debe repetir una o más materias, lo que repercute en el poco o nulo control acerca de la información correspondiente de cada estudiante que se encuentra en esta situación.

La problemática presente en la gestión de la educación por las razones mencionadas anteriormente, han incrementado su complejidad y a su vez, ha tenido que dar respuesta a otras adversidades que se han generado. Estas modificaciones han provocado una serie de desajustes tanto para la población estudiantil como para el personal docente, docente administrativo y administrativo, alterando la estructura de la gestión institucional. Lo anterior, se convierte en un importante reto para la administración de la educación, actualmente.

De acuerdo con la problemática mencionada y el contexto educativo, surgen diferentes interrogantes sobre la aplicación de las Normas reguladoras de la promoción y repitencia dentro del sistema educativo público costarricense, con el fin de analizar este tema desde la gestión del Colegio Técnico Profesional de Limón, circuito 01, de la Dirección Regional de Limón. Al respecto, se plantea la siguiente interrogante: ¿Cómo incide la gestión administrativa en el aprovechamiento de las normas reguladoras de la promoción y repitencia en el sistema educativo público costarricense? 
Esta investigación ofrece insumos, datos y resultados relevantes para mejorar la administración de la educación, en torno a la aplicación de las normas reguladoras de la promoción y repitencia dentro del sistema educativo público costarricense. Por tanto, es importante considerar que, si se implementan de forma eficiente, se puede llevar un mejor control de las actividades a desarrollar en las instituciones educativas, con el fin de proyectar y organizar de mejor manera las estrategias, para el fiel cumplimiento de los fines de la educación costarricense. Además, el estudio busca ofrecer aportes a la gestión para el mejoramiento de la calidad de la educación que se brinda a la comunidad educativa, específicamente a la población estudiantil.

Asímismo, esta investigación permite brindar aportes en el área administrativa de las organizaciones educativas, en cada una de sus distintas funciones, debido a los diversos factores del campo educativo y administrativo que se interrelacionan en la aplicación correcta de las normas relacionadas con la educación del país, de la cual es el profesional en el campo de la administración de la educación, el responsable de velar para que las regulaciones acerca de la evaluación estudiantil se desarrollen según el Reglamento de Evaluación de los Aprendizajes vigente $y$, de esta forma, se puedan planear, organizar y controlar las estrategias a desarrollarse en el curso lectivo para el logro de los objetivos institucionales y de la educación costarricense.

Para ello, se estructuran los siguientes objetivos, general y específicos:

\section{Objetivo general}

- Analizar la gestión del Colegio Técnico Colegio Técnico Profesional de Limón, circuito 01, de la Dirección Regional de Limón, en torno a la aplicación de las normas reguladoras de la promoción y repitencia dentro del sistema educativo público costarricense.

\section{Objetivos específicos}

- Describir los efectos que ha generado la aplicación de la normas de promoción y repitencia del sistema educativo costarricense en los procesos de planeación de la gestión institucional.

- Identificar las estrategias administrativas y educativas desarrolladas por la gestión para coordinar la puesta en práctica de las normas de promoción y repitencia vigente.

- Identificar las estrategias administrativas y educativas desarrolladas por la gestión para dirigir la puesta en práctica de las normas de promoción y repitencia vigente. 
- Reconocer las estrategias administrativas y educativas que ejerce la gestión de la institución para controlar los procesos que requiere la aplicación de las normas de promoción y repitencia vigente.

- Determinar la metodología que ha desarrollado la gestión para evaluar la aplicación de las normas de promoción y repitencia vigente.

- Identificar las ventajas y desventajas que ha generado la aplicación de las normas de promoción y repitencia a nivel institucional.

Con la finalidad de desarrollar los objetivos propuestos, se seleccionó el Colegio Técnico Profesional de la provincia de Limón, en Costa Rica, debido a la población tan diversa que posee esta institución, el número de estudiantes que se encuentran repitiendo una o más materias y la problemática que se ha presentado en torno a la aplicación de las normas de promoción y repitencia.

El Colegio Técnico Profesional de Limón pertenece al Circuito 01 de la sede regional del Ministerio de Educación Pública, fundado en el año 1967 como una parte del Colegio Diurno de Limón y luego, independizado de este último en respuesta a las necesidades presentes en la sociedad. Debido al crecimiento que ha tenido a través de los años, el Colegio Técnico Profesional de Limón ha sido categorizado como dirección tres², ya que tiene la capacidad de albergar alrededor de 1500 estudiantes.

\section{Marco referencial}

De acuerdo con lo planteado por el MEP (2011), el sentido de la reforma al Reglamento de evaluación de los aprendizajes surge para "mejorar el rendimiento y el aprendizaje de los estudiantes" (p.3). Esta normativa y reforma nace con el propósito de evitar ciertas situaciones que, de alguna manera, se encuentran perjudicando al estudiantado en los centros educativos, por lo que tiene la finalidad de apoyar y promover el mejoramiento del rendimiento académico de cada estudiante dentro del sistema educativo; además de proveer los recursos necesarios para que la niñez y la juventud asistan a recibir lecciones sin preocupaciones externas y sin que otros factores, como su situación económica, afecten este rendimiento.

Las normas propuestas en ese sentido, consisten en que la población estudiantil pueden aprobar el curso lectivo, obteniendo la nota mínima de aprobación en su calificación ponderada anual, la

\footnotetext{
Se designa una numeración a la Dirección por la capacidad de matrícula que poseen las instituciones educativas.
}

\section{(c) (1) (9)}


subdivisión de los períodos por porcentajes, presentar pruebas de convocatoria hasta en cuatro asignaturas y cursar materias del nivel inmediato superior al que reprobó, mientras repite la asignatura que tienen pendiente del nivel que cursa.

Estas normas permiten a toda la población estudiantil adolescente que ha desertado del sistema educativo, reanudar su motivación para continuar con sus estudios, pudiendo llevar las materias que perdieron sin la presión de volver a recibir materia que ya fue aprobada anteriormente.

\section{Marco teórico}

\section{Educación}

La educación es un aspecto vital del desarrollo social del ser humano, ya que mediante ésta logra integrarse de forma adecuada a la sociedad y así, mejorar su calidad de vida. En ese sentido, Vargas (2008) señala que "la educación sigue siendo un valor histórico para la sociedad costarricense y que el sistema educativo es valorado como un elemento de realización personal y profesional para los ciudadanos" (p. 2).

Otra definición de educación, dada por Zayas y Rodríguez (2010), indica que “... no es un proceso restringido a los procesos escolares. Es un fenómeno social extendido más allá de ellos y de las formas oficiales relacionadas con los mismos" (p. 4). De esta manera, el entorno familiar es el que provee los primeros conocimientos de comportamiento social; es ahí, donde cada persona va aprendiendo a ser parte de la sociedad en la cual está inmerso. Es así, que la educación es un proceso por medio del cual se transmite la información de conocimientos, con la finalidad de dar continuidad al desarrollo general de la población.

El ser humano, como ser social, se ha reunido con sus iguales para conformar organizaciones diseñadas con el fin de brindar o prestar un servicio; éste puede que se realice con fines de lucro o sin ellos. Toda organización constituida cuenta con una persona que está encargada de la toma de decisiones para generar eficiencia y eficacia en los servicios que brindan, aunado al logro de los objetivos para los que fue creada. De ahí, que las organizaciones educativas requieran de procesos de administración que orienten el desarrollo de servicios educativos que se ofrecen a la sociedad.

\section{Administración de la educación}

De acuerdo con Chiavenato (2006), la administración "no es un fin en sí mismo, sino un medio de lograr que las cosas se realicen de la mejor manera, al menor costo y con la mayor eficiencia y eficacia" (p. 13). Es por lo anterior, que en el sector educativo se inserta el concepto de administración de la educación, de tal forma que se ayude a mejorar la planificación, organización, dirección y control que se requiere para elevar la calidad de la educación. 
Por ello, la administración y la educación, logran formar el engranaje perfecto que permite desarrollar el proceso educativo de forma adecuada. La administración de la educación es la encargada de efectuar las gestiones necesarias por medio de las cuales se logran los objetivos trazados en una institución educativa. Al respecto, Garbanzo y Orozco (2007), indican que:

(...) la administración aplicada al campo de la educación conlleva la responsabilidad de conducir adecuadamente los procesos de gestión educativa en los distintos niveles y modalidades de la educación articulada a un fuerte enfoque pedagógico, para poder alcanzar el desarrollo de los aprendizajes con altos parámetros de calidad. (p. 97)

En razón de lo anterior, es la administración de la educación la encargada de definir los medios por los que se solventan las necesidades presentes en el campo educativo, de manera que se cumpla con los estándares definidos para ofrecer una educación de calidad, comprendida como los resultados obtenidos con la mayor eficiencia y eficacia, lo mejor de lo mejor.

Debido al modelo de organización lineal ${ }^{3}$ del Ministerio de Educación Pública, es necesario que todas las directrices emanadas por esta entidad, sean difundidas de tal modo que se llegue a coordinar entre todas las instituciones educativas del territorio nacional; éstas a su vez, deben ser llevadas a cabo por cada profesional que ejerce la gestión administrativa de la educación, incorporándolos al contexto de su zona laboral y utilizando las estrategias que se consideren necesarias para el logro de los objetivos propuestos.

El Consejo Superior de Educación de Costa Rica (1994) menciona en el documento titulado Política Educativa Hacia el Siglo XXI que "la administración central y regional es el tamiz por el que forzosamente pasan todas las acciones tendientes a desarrollar los mandatos de la política educativa" (p. 4). Todas las reformas propuestas por el MEP, por tanto, deben ser ejecutadas por los profesionales en administración de la educación de cada centro educativo del país. En este tipo de organización, siempre se van a encontrar jerarquías, es decir, distintos administradores por nivel en la organización. En cuanto a la organización del MEP, se tiene que el profesional que actúa como administrador primario es el Ministro de Educación, luego los directores regionales y en las instituciones educativas, propiamente los profesionales en administración de la educación y el personal docente.

A lo interno de cada institución educativa, las personas gestoras organizan su trabajo por departamentos y comités, los que a su vez, tienen la responsabilidad de confeccionar objetivos

\footnotetext{
Para Chiavenato (2006), "organización lineal significa que existen líneas directas y únicas de autoridad y responsabilidad entre superior y
} subordinado" (p. 60). 
específicos en relación con todos los programas que envía el MEP para ser implementados en las organizaciones educativas. De esta forma, el profesional que desarrolla la gestión debe coordinar las estrategias que se van a ejecutar para el logro de dichos objetivos. Según Veciana (1998), coordinar "significa ordenar y armonizar las partes de un todo unitario" (p. 54).

De ahí, que los profesionales en administración de la educación, en su proceso de gestión institucional, debe ordenar y armonizar al personal que tiene a su cargo, con la finalidad de distribuir las estrategias que se desean llevar a cabo; de tal manera, que se delegue el trabajo correspondiente entre los miembros del personal. Esta designación debe tomar en cuenta la escogencia del personal idóneo para cumplir con el trabajo, tener presente las fortalezas y debilidades del mismo, en función de la asignación de tareas de acuerdo con las estrategias y capacidades que poseen.

Los departamentos, en ese sentido, son los encargados de atender asuntos específicos emanados por la persona que ejerce un puesto superior para el alcance de los objetivos; así también, los profesionales de la administración de la educación que desempeñan la gestión pueden designar comités, los cuales son agrupaciones de personas establecidas en la organización educativa, para el cumplimiento de funciones técnicas; es decir, se encargan de analizar ciertos asuntos en los que tienen más experticia y especialización que profesionales que ejercen el puesto de dirección en los centros educativos.

Inmerso en el área educativa, el control administrativo es una de las cuatro funciones que debe desarrollar todo profesional en administración de la educación, sin importar la institución en la que se encuentre laborando, ni el nivel jerárquico que se encuentre administrando. La finalidad del control, de acuerdo con Chiavenato (2006) es entonces, "asegurar que los resultados de aquello que se planeó, organizó y dirigió se ajuste tanto como sea posible a los objetivos establecidos" (p. 151). El control es la función por medio de la cual, los profesionales en administración de la educación pueden determinar el logro o no de los objetivos propuestos en la institución.

Desde esta perspectiva, se deben considerar las fases del control expuestas por Chiavenato (2006), las cuales se citan a continuación:

- Establecimiento de estándares

- Observación del desempeño

- Comparación del desempeño con el estándar establecido

- Acción correctiva 
Con respecto a las fases anteriormente mencionadas, en cada proceso de la administración de la educación, se deben definir los criterios por medio de los cuales se va a establecer el control de algún programa, normativa o proyecto. Para ello, se toman en cuenta los objetivos establecidos en la gestión del proceso de planeación y definición de los criterios respectivos con los que se va a medir el desempeño de lo que se desea controlar y por último, diseñar la acción correctiva que se va a implementar en el caso que no se esté cumpliendo con lo esperado.

Son los profesionales en administración de la educación, quienes en cada institución educativa, se encargan de establecer una serie de estrategias que le permitan identificar el cumplimiento de los objetivos propuestos por el MEP, en el nivel macro y definir objetivos nuevos de acuerdo con su contexto, en la planificación realizada previamente, de tal forma que puedan efectuar las modificaciones necesarias en caso de requerirlas. Para un mejor entendimiento del concepto de estrategia, Hax y Majluf (2004), la definen como el "modo de dar explícitamente forma a las metas y los objetivos de largo plazo de la organización, definiendo los principales programas de acción necesarios para alcanzar los objetivos y desplegando los recursos necesarios" (p. 25). Las organizaciones educativas, por tanto, deben establecer estrategias de control que les permitan tener conocimiento de las necesidades que se presentan y a su vez, verificar el desempeño de la misma.

En ese sentido, el MEP (2011) ha girado ciertas directrices en relación con el control de los procesos que se desarrollan en la educación pública y privada, lo que ha permitido, en poca o gran medida, hacer las modificaciones que corresponden o el reforzamiento de los mismos, propiciando el logro de los objetivos institucionales. Al respecto:

(...) se entenderá que un efectivo sistema de control interno es aquel que apoya y propicia el logro de los objetivos del Centro Educativo y del Ministerio en General, es decir un sistema de control interno parte de la existencia de objetivos y metas claras y que estas sean del conocimiento de todo el personal docente y administrativo. (p. 2)

De acuerdo con la cita anterior, todo profesional en administración de la educación debe establecer ciertos objetivos acerca de cada uno de los proyectos y programas sociales, emanados por el MEP para su ejecución, como por ejemplo el programa avancemos, el cole en nuestras manos y otros, en función de evitar la deserción estudiantil en los centros escolares y el sistema educativo, en general. 


\section{Gestión de la educación}

La gestión es considerada como un proceso cuya responsabilidad recae en la figura de los profesionales en administración presentes en las organizaciones educativas, quienes ejercen la función de dirección, en compañía de un equipo de personas con las cuales trabajan de forma conjunta e integral para el logro de los objetivos institucionales propuestos, de forma eficaz y eficiente. Por ello, la gestión puede entenderse como un proceso activo de la administración de la educación, en el que se utilizan las mismas funciones administrativas para su ejecución y, de esta manera, favorecer la retroalimentación de la organización educativa.

En ese sentido, Venegas (2011), se refiere a la gestión de la siguiente manera:

Conjunto deacciones articuladas entre sí, que posibilitan la consecución de la intencionalidad de la institución, como aquel proceso que articula las múltiples instancias de la vida escolar con una sola dirección, la de lograr aprendizajes significativos en los alumnos. (p. 5)

De esta manera, la gestión es muy importante para el desarrollo de todas las funciones de la administración de la educación, puesto que permite un manejo adecuado de los recursos y de los medios por los cuales se van a desarrollar las estrategias que los profesionales en administración de la educación, en su gestión, consideren necesarios para mejorar el servicio educativo que se brinda.

\section{La evaluación de las organizaciones en el marco educativo.}

El proceso de evaluación es muy amplio y abarca distintas áreas en el campo de la educación. Ésta se implementa no solo con la población estudiantil, sino que se aplica en caso de requerir una valoración del personal docente, del desarrollo curricular, el desempeño organizacional y de los programas institucionales, en pro del mejoramiento del servicio y otros. La evaluación puede ser ejecutada de distintas formas o metodologías por parte de la persona que cumple la función evaluadora, según sea el caso.

El proceso evaluativo se puede manifestar de distintas maneras. De ahí, que la evaluación puede ser utilizada como un diagnóstico de la implementación de algún programa instaurado, puede tener un énfasis formativo, de tal manera que los resultados que se obtengan puedan generar ciertos cambios en pro del aprendizaje o para la modificación de ciertas estrategias que se estén aplicando, que no generan los insumos deseados, entre otros. La metodología de 
evaluación es la forma por medio de la cual se lleva a cabo el proceso evaluativo, por lo que es la manera que determina el profesional que desarrolla la función evaluadora para ejecutar la evaluación del objeto deseado.

\section{Rendimiento académico}

El rendimiento académico de la población estudiantil es un aspecto relevante que influye, directamente, en cada estudiante y sus familias, por lo que éste puede interferir en las decisiones del núcleo familiar de forma positiva pero, a la vez, puede hacerlo de forma negativa y permanentemente.

En relación con lo anterior, Rodríguez y Gallegos (1992), opinan que el rendimiento académico refiere:

(...) un sistema de interacciones entre factores aptitudinales, familiares, relaciones profesor-alumno, alumno-compañeros, método de enseñanza..., considerados cada uno de ellos no solo como sumandos, sino también como elementos o variables que se influyen mutuamente dos a dos y, a su vez, entre los tres. (p.14)

Por lo anterior, se puede relacionar el rendimiento académico con el contexto en el cual se desenvuelve la población estudiantil, sus expectativas, las de su familia, los recursos con los que cuenta para desarrollar su potencial, la situación socio afectivo, económico y otros componentes. El rendimiento académico se encuentra directamente relacionado con los métodos y estrategias con los que se atiende al estudiantado en la institución en la que se encuentran y los esfuerzos que la población estudiantil haya manifestado en el proceso para salir adelante, sin darle mucha relevancia a los objetivos del proceso enseñanza-aprendizaje.

En muchos hogares costarricenses, cuando el rendimiento de los hijos e hijas es bajo, los padres y madres de familia toman la decisión de retirarlos del sistema educativo formal y optan por que se quede en casa colaborando con las labores del hogar, o si poseen la edad necesaria para obtener un empleo, les sugieren buscarlo para ayudar con las necesidades que se presentan; a este fenómeno, se le conoce como deserción escolar. Ante las diversas situaciones que se presentan en torno a la deserción estudiantil en los centros escolares, el MEP pretende disminuir los índices de este en el sistema educativo costarricense con diferentes estrategias, para lo cual promueve el desarrollo de reformas a las normas educativas existentes. 


\section{Deserción escolar}

Para definir el concepto de deserción escolar, es relevante considerar la opinión de Cordero (2008), quien la señala como "el abandono de los estudios y rezago escolar es producto de un conjunto de causas multidimensionales, las cuales se relacionan, entre otros con las condiciones económicas, académicas y metodológicas, pero también culturales e ideológicas" (p. 4).

En algunas ocasiones, el personal docente puede identificar comportamientos en los que incurre la población estudiantil que piensa desertar del sistema educativo. De esta manera, las razones por las cuales el estudiantado desertan de éste, son variadas, dentro de las cuales se encuentran situaciones que suceden dentro de la institución educativa que afectan tanto al estudiante como a su contexto social. A lo interno del centro educativo, cuando se inicia la falta a lecciones por parte de la población estudiantil, sin presentar una justificación válida; cuando sus calificaciones decaen de forma drástica y no se interesan por mejorarlas, son llamadas de atención para que el personal docente preste atención y traten de motivar a aquellos estudiantes que se encuentran en riesgo de desertar, con el fin de que no abandonen sus estudios ni se generen nuevas acciones que afecten su contexto social por la inseguridad que se vive en algunas zonas del país, la situación económica del hogar, entre otras.

En la educación costarricense, el porcentaje de estudiantes que desertan del sistema educativo formal es elevado. Estas cifras han sufrido una transformación y tienden a la baja, según el último Informe del Estado de la Educación (CONARE, 2011), el cual menciona que "la deserción se redujo del 11,6\% en el 2006 al 9,5\% en el 2009" (p. 125). Lo anterior, gracias a que el gobierno ha implementado, en los últimos años, una serie de estrategias para evitar la deserción, procurando disminuir los porcentajes expresos en la educación costarricense.

\section{La calidad de la educación}

A través de los años, el gobierno costarricense ha procurado ampliar la cobertura que tiene la educación en el territorio nacional; además de elevar la calidad del personal que tiene laborando en las distintas áreas y puestos donde se brinda el servicio educativo, lo que incide, a su vez, en el mejoramiento de la calidad educativa en las regiones. La transmisión de la historia cultural y el desarrollo de todas las potencialidades de las personas, es parte de las funciones principales para brindar una educación de calidad, con el fin de satisfacer las necesidades de la mayor cantidad de integrantes de la población nacional. El hecho de que todos los habitantes de la nación tengan acceso a la educación 
que se brinda en las mejores instituciones educativas del área metropolitana, es una de las condiciones esenciales que evidencian el alcance de un nivel elevado de calidad en cuanto a la educación del país.

De ahí, que una educación adecuada y pertinente permita brindar todos los apoyos necesarios para que ésta sea acorde con los objetivos propuestos en la educación costarricense.

La educación es un proceso que lleva a cabo una serie de funciones que tienen como finalidad el desarrollo de la nación, es por esto que se debe definir lo que es un proceso educativo de calidad. Al respecto, para el Consejo Superior de Educación de Costa Rica (1994), en la Política Educativa Hacia el Siglo XXI, refiere que:

El proceso educativo de calidad involucra el proceso y los resultados de acuerdo con los Fines. Además, ofrece igualdad de oportunidades para el éxito a quienes participan en él y propone una oferta coherente con las necesidades, problemas y aspiraciones de los participantes en relación con su entorno. (p. 7)

La relevancia de mejorar la calidad en el servicio educativo en la sociedad actual, es de vital importancia para estar acordes con el nivel de competitividad existente en el área educativa, en relación con la dinámica escolar de otros países de América Latina e incluso, con países que son considerados como potencias mundiales.

\section{Metodología}

La investigación tiene un enfoque exploratorio-cuantitativo, ya que acerca de este tema no se han efectuado otras investigaciones que tengan relación directa con el estudio.

La población participante de esta investigación, la conforman 187 estudiantes del Colegio Técnico Profesional de Limón, quienes se encuentran repitiendo asignaturas, distribuidos en los niveles desde sétimo año a duodécimo año. También, el personal docente encargado de ejecutar la parte administrativa en la implementación de la normativa de promoción y repitencia vigente, el profesional en administración de la educación y el total de personal docente (98 personas) que imparten diferentes asignaturas técnicas, académicas y que poseen al menos un estudiante que se encuentra repitiendo la asignatura que imparten.

La siguiente tabla, resume la población participante en el estudio. 
Tabla 1

Distribución de la población del estudio

\begin{tabular}{lc}
\hline Población del estudio & $N^{\circ}$ de participantes \\
\hline Directora & 1 \\
Profesional encargado & 1 \\
Docentes & 98 \\
Sétimo año & 25 \\
Octavo año & 47 \\
Noveno año & 49 \\
Décimo año & 36 \\
Undécimo año & 20 \\
Duodécimo año & 6 \\
\hline Total & 283 \\
\hline
\end{tabular}

Fuente: Jobson, 2012. Elaboración propia.

La información fue recolectada por medio de un cuestionario dirigido al personal docente que labora en la institución con estudiantes que se encuentran repitiendo una o más asignaturas y otro, aplicado a la población estudiantil que cumple con el requisito de estar repitiendo al menos una asignatura para ser parte de este estudio. Además, se utilizó la técnica de entrevista dirigida para la dirección del Colegio Técnico Profesional de Limón, así como para el profesional encargado de la implementación de las normas reguladoras de la promoción y repitencia dentro del sistema educativo público costarricense.

Par orientar el desarrollo de la investigación, se definieron seis categorías de análisis para el estudio de los objetivos que son:

- Procesos de planeación de la gestión institucional

- Estrategias para coordinar

- Estrategias para la dirección

- Estrategias de control de procesos

- Metodología de evaluación

- Las ventajas y desventajas que ha generado la aplicación de las normas reguladoras de la promoción y repitencia dentro del sistema público costarricense en la gestión institucional 


\section{Análisis de la información}

A continuación, se exponen los principales resultados obtenidos en la investigación, de acuerdo con las categorías de análisis expresas anteriormente, con el fin de estructurar la información de manera precisa, conforme a los objetivos establecidos.

\section{Procesos de planeación de la gestión institucional}

En cuanto al proceso de planeación que realiza la gestión del Colegio Técnico Profesional de Limón, no se establecen objetivos específicos para la implementación de las normas reguladoras de la promoción y repitencia dentro del sistema educativo público costarricense.

Al respecto, es importante tomar en cuenta lo indicado por Daft y Marvic (2006), al referirse a la planeación como "determinar las metas de la organización y definir los medios para lograrlos" (p.146). De esta forma, queda clara la necesidad de que la gestión cumpla con los propósitos de la institución, por lo que se deben crear objetivos que delimiten el rumbo por el cual se quiere llevar cada uno de los programas, proyectos y campos de acción de la educación, con los que debe cumplir. Al no haber ningún objetivo programado por medio del cual se ejecute la implementación de las normas de promoción y repitencia, no se tiene un panorama claro de lo que se quiere obtener de esa acción en concreto.

Los hallazgos manifiestan la importancia que posee la administración de la educación en la gestión de las instituciones educativas de secundaria, en cuanto a la aplicación de las normas reguladoras de la promoción y la repitencia dentro del sistema educativo público costarricense, siendo la encargada de guiar y dar las pautas a seguir, con el fin de cumplir con los fines de la educación costarricense.

\section{Estrategias para coordinar}

En cuanto a las estrategias para coordinar la implementación de las normas reguladoras de la promoción y repitencia dentro del sistema educativo público costarricense, en las organizaciones educativas, se presentan los siguientes hallazgos.

Una vez realizada la triangulación de la información obtenida por la población participante, se puede determinar que la mayoría del personal docente no tiene conocimiento de la manera como se maneja la implementación de las normas de promoción y repitencia dentro del sistema público costarricense, por falta de coordinación en la gestión. La información en manos de pocas personas y además, la gestión institucional no comunica, de forma asertiva, los datos básicos que se deben manejar sobre la normativa vigente; la implementación se está efectuando 
solamente con la información general que dio el MEP para que se iniciara en todos los colegios del territorio nacional.

Es importante, en este sentido, considerar que la gestión administrativa debe ser la encargada de implementar cada una de las propuestas designadas por el MEP, para que se llegue aplicar en todas las instituciones educativas del país. La gestión de cada institución debe realizar los procesos que sean necesarios para una implementación adecuada de las normas ya establecidas, de tal manera que se apliquen de forma oportuna, según las necesidades que se presenten en cada institución.

\section{Estrategias para la dirección}

En cuanto a las estrategias para la dirección en la implementación de las normas reguladoras de la promoción y repitencia dentro del sistema educativo público costarricense, se exponen los siguientes resultados.

La población consultada en el tema de estrategias de dirección, en su mayoría expresan el desconocimiento acerca de las acciones que se realizan con la finalidad de dirigir al personal en relación con la aplicación de la normativa.

Las estrategias, según Ugalde (2011), son "como un plan o curso de acción, un modelo o patrón de decisiones que determina y revela los objetivos empresariales, es la forma en el gerencia percibe el entorno competitivo y ubica a la organización dentro de ese ambiente" (p. 431).

De acuerdo con la definición anterior, se dificulta establecer una concepción clara sobre las estrategias de dirección, ya que no se dan objetivos por medio de los cuales se conozca el camino por el que se quiera guiar al personal. Las respuestas obtenidas en las que se expresa la opinión de la población participante, se evidencia la falta de estrategias de dirección implementadas por la gestión del centro educativo en estudio, en relación con las normas reguladoras de la promoción y repitencia.

\section{Estrategias de control de procesos.}

En relación con las información recopilada a través de las técnicas e instrumentos aplicados, se evidencia que el control de la implementación de las normas reguladoras de la promoción y repitencia dentro del sistema público costarricense, no se está ejerciendo de forma efectiva en la institución en estudio, de acuerdo con lo que establece el Ministerio de Educación Pública (2011), en cuanto al control, el cual: 
(...) se entenderá que un efectivo sistema de control interno es aquel que apoya y propicia el logro de los objetivos del Centro Educativo y del Ministerio en General, es decir un sistema de control interno parte de la existencia de objetivos y metas claras y que estas sean del conocimiento de todo el personal docente y administrativo. (p. 2)

Para que se lleve a cabo un efectivo sistema de control, es importante el establecimiento de objetivos específicos previamente, acerca de las normas reguladoras de la promoción y repitencia dentro del sistema educativo público costarricense, de tal forma que al efectuar los instrumentos para controlar la aplicación de esta normativa, se establezcan criterios con base a los objetivos propuestos con antelación.

\section{Metodología de evaluación}

Con respecto a la información obtenida mediante la aplicación de técnicas e instrumentos para la recolección de datos, se determina que la gestión del Colegio Técnico Profesional de Limón no ha aplicado ningún método de evaluación por medio del cual se tenga conocimiento de los efectos que ha generado la implementación de las normas de promoción y repitencia vigente en el contexto institucional, con más precisión en la gestión institucional.

Al respecto, Chavarría y Borrel (2003) mencionan que la evaluación "se ha identificado con medición, calificación, responsabilidad, estimación o apreciación" (p. 50). De acuerdo con lo anterior, la gestión de la institución en estudio no ha desarrollado ninguna estrategia con el propósito de medir, apreciar o estimar la implementación de la normativa de promoción en la institución, por lo que no existe una metodología en ese sentido.

A partir de la información obtenida por la población consultada sobre la existencia de un plan de contingencia referido a la implementación de las normas de promoción vigentes, se determina que no existe un plan de ese tipo, así como no se tomó en consideración la implementación de las normas de promoción y repitencia en el plan quinquenal, ni se ha efectuado un plan remedial que contemple la aplicación de las normas. Por ello, la gestión institucional no ha realizado el proceso administrativo necesario con respecto de la implementación de las normas de promoción y repitencia vigentes que debiera considerarse para el mejoramiento de la calidad de la educación y evitar la deserción estudiantil de los centros educativos.

En ese sentido, Chiavenato (2006) define el proceso administrativo como el "conjunto de funciones administrativas o elementos de la administración, planeación, organización, dirección y control. Es un proceso cíclico y sistémico" (p. 157). Según la definición dada, en el Colegio 
Técnico Profesional de Limón, no se realiza un adecuado proceso administrativo en relación con la implementación de las normas reguladoras para la promoción y repitencia en el sistema educativo público costarricense.

\section{Ventajas y desventajas de la aplicación de las normas reguladoras de la promoción y repitencia dentro del sistema público costarricense en la gestión institucional}

Las normas reguladoras de la promoción y repitencia dentro del sistema educativo público costarricense no son vistas de forma negativa en su totalidad, por el contrario, las modificaciones que se efectuaron al Reglamento de Evaluación de los Aprendizajes brinda una mayor oportunidad a la población estudiantil de permanecer en los centros educativos, de tal manera que avancen en su formación, a pesar de haber perdido una o varias asignaturas. Sin embargo, la problemática se presenta en aspectos administrativos, por lo que es relevante para la gestión de la institución, tener un conocimiento más preciso de la cantidad de estudiantes que se encuentran repitiendo materias, con el fin de ubicarlos en la sección que le brinde mejores oportunidades sin que esto signifique que todos sean ubicados en la misma sección, ya que por las particularidades que presenta cada caso no debería ser así.

Es positivo que la mayoría de la población estudiantil manifieste que, con la aplicación de estas normas, se da la oportunidad a más jóvenes de tener acceso a la educación si en algún momento desertaron. De de esta manera, se motivan y continúan con el proceso enseñanzaaprendizaje, a pesar que el resto de la población piense de diferente manera.

De acuerdo con la información brindada por toda la población participante en el estudio, en la categoría de ventajas y desventajas, es evidente la necesidad de diseñar un proceso de planeación en el que se establezca un comité integrado por personal docente, docente administrativo y la persona encargada de la implementación de las normas reguladoras de la promoción y repitencia dentro de sistema educativo público costarricense, con el fin de crear objetivos y estrategias específicas para que se tenga un mejor control de la aplicación de esas normas en la institución.

La gestión de la educación, en ese sentido, debe aplicar un proceso administrativo para implementar las normas reguladoras de la promoción y repitencia dentro de sistema educativo público costarricense, que concuerde con la definición dada por Chiavenato (2006), quien menciona que el procedimiento administrativo es un "conjunto de funciones administrativas o elementos de la administración, planeación, organización, dirección y control. Es un proceso cíclico y sistémico" (p. 157). De esta manera, si la gestión aplica el proceso administrativo antes mencionado con respecto a las normas de promoción y repitencia vigentes, se puede tener un panorama más claro de lo que la gestión pretende y se planean mejor las estrategias por medio de las cuales se puede llegar a mejorar la forma como están siendo implementadas hasta este año. 
Las normas han incidido en gran medida en el rendimiento académico de la población estudiantil, puesto que, de acuerdo con la información obtenida, ha creado una cantidad de efectos negativos, tanto en el proceso de administración de la educación como en el estado anímico del estudiantado que se encuentran repitiendo alguna asignatura.

\section{Conclusiones}

Conforme los hallazgos encontrados, en función de los objetivos establecidos para la investigación, se presentan las siguientes conclusiones:

Se evidencia la carencia de un plan confeccionado por la gestión de la educación del centro educativo, por medio del cual se hayan definido objetivos o metas diseñados con la finalidad de una implementación de las normas reguladoras de la promoción y repitencia dentro del sistema educativo público costarricense, de manera pertinente.

En ese sentido, el plan quinquenal que se encuentra vigente en el Colegio Técnico Profesional de Limón fue confeccionado en el año 2009 y no contempla objetivos que tengan relación con alguna meta propuesta acerca de estas normas, ya que no fueron tomadas en cuenta a la hora de confeccionarlo, a pesar de los cambios al Reglamento de Evaluación de los Aprendizajes, efectuados en el año 2008. Tampoco se ha promovido la confección de un plan remedial que contemple los objetivos que atiendan esta normativa con el transcurso de los años.

Además, no se ha realizado una contextualización de las normas reguladoras de la promoción y repitencia dentro del sistema educativo público costarricense, de acuerdo con las necesidades que posee la población estudiantil, el personal docente y la comunidad educativa, en general.

La administración de la educación del centro educativo, no realiza estrategias con la finalidad de coordinar actividades que tengan relación con la implementación de las normas reguladoras de la promoción y repitencia dentro del sistema educativo público costarricense. Al respecto, se tiene desconocimiento de los recursos que se necesitan para la aplicación de esa normativa, de tal manera que se lleve a cabo de forma eficaz y eficiente.

El trabajo de ejecutar la implementación de las normas está recargado en una sola persona que también tiene responsabilidades como docente de la institución; no se coordinan los recursos para que todo el personal docente y administrativo trabaje en forma conjunta y se logre así, una mejor implementación de la normativa.

Las estrategias diseñadas para dirigir al personal con respecto a la implementación de las normas reguladoras de la promoción y repitencia dentro del sistema educativo público 
costarricense, son conocidas por muy pocas personas que laboran en la institución; solo se dan directrices de trabajo a la persona encargada de la implementación de las normas en mención. Al personal docente, solo se le pide cumplir con las funciones que les corresponde, de acuerdo con el Reglamento de Evaluación de los Aprendizajes.

En la institución participante del estudio, no se da un eficiente sistema de control interno. Las normas reguladoras de la promoción y repitencia dentro del sistema educativo público costarricense se aplican en la institución, pero son pocas o nulas las actividades que se efectúan para llevar un control de la implementación de las mismas. La persona que maneja la mayor información con respecto a las normas, es la encargada de la implementación y no aplica ningún tipo de control.

Debido al poco tiempo que lleva la personal profesional en administración de la educación como directora del Colegio Técnico Profesional de Limón, los integrantes del personal que son parte de la gestión institucional, han debido ajustarse al cambio y en algunos casos, fueron sustituidos por otras personas. Lo anterior, ha provocado un reacomodo en la estructura institucional y en la revisión de objetivos que se habían plasmado en un plan quinquenal deficiente, pues no incluían algunas áreas y necesidades organizacionales.

Asimismo, la gestión de la institución en estudio no cuenta con estrategias establecidas para establecer el control de los procesos que se relacionan, directamente, con las normas reguladoras de la promoción y repitencia dentro del sistema público costarricense, por lo que no se tiene el control de la implementación y solo se aplican las directrices establecidas por el Ministerio de Educación Pública, desde la publicación de la normativa.

La gestión institucional no ha implementado ningún método de evaluación que evidencie el desempeño de las normas reguladoras de la promoción y repitencia dentro del sistema educativo público costarricense en el centro educativo, por lo que la institución no puede efectuar una evaluación de la implementación de la normativa. No se establecieron objetivos por medios de los cuales se diera la implementación de las normas, ni se ha construido ningún instrumento al respecto, para medir los alcances que ha tenido la aplicación de la normativa en estudio.

En el Colegio Técnico Profesional de Limón, de acuerdo con los hallazgos de la investigación, no se han desarrollado actividades que conlleven la implementación del proceso administrativo pertinente a las normas reguladoras de la promoción y repitencia dentro del sistema educativo público costarricense, por lo que la gestión de la institución debe enfocarse en eso.

La implementación de las normas en mención, ha generado algunas ventajas para la población estudiantil que se encuentra repitiendo alguna materia pero, a su vez, desventajas para todos los protagonistas del proceso de enseñanza-aprendizaje. De los sectores que presentan más problemas en la institución, en relación con la implementación de las normas 
reguladoras para la promoción y repitencia, están: la parte administrativa y curricular, la ubicación del estudiantado, la confección de horarios, el seguimiento que hay que darle a la población estudiantil que se encuentran repitiendo, otros.

Para atender esas problemáticas, la gestión de la institución ha diseñado varias estrategias para rectificar algunas de las situaciones que se están presentando en la actualidad. Sin embargo, se puede concluir que la institución en estudio necesita reforzar el trabajo que se realiza para mejorar el servicio que está prestando, ya que no se están realizando todas las acciones pertinentes que se requieren para la aplicación, efectiva y eficiente, de la implementación de las normas de la promoción y repitencia vigentes de forma adecuada. En ese sentido, no fueron tomadas en cuenta a la hora de efectuar el plan institucional, por lo que no se está cumpliendo con las disposiciones dadas por el Ministerio de Educación Pública.

\section{Referencias}

Asamblea Constituyente de la República de Costa Rica. (1949). Constitución Política de la República de Costa Rica. Recuperado de http://www.constitution.org/cons/costaric.htm

Asamblea Legislativa de la República de Costa Rica. (1957). Ley Fundamental de Educación $N^{\circ} \quad 2160$. Recuperado de http://www.mep.go.cr/CentroDelnformacion/DOC/ leyfundamental-285200810525.pdf

Castells, M. (2005). La era de la información: económica, sociedad y cultura (Vol. I). México: Siglo veintiuno.

Chavarría, X. y Borrel, E. (2003). Evaluación de centros educativos. Aspectos nucleares. España: Editorial UOC.

Chiavenato, I. (2006). Introducción a la teoría general de la administración. México: McGraw-Hill.

Cordero, T. (2008). La opinión de un grupo de docentes sobre la deserción escolar . Explorando sobre sus actuaciones en el contexto institucional. Revista Actualidades Investigativas en Educación, 8(3), 1-33. Recuperado de http://redalyc.uaemex.mx/redalyc/ pdf/447/44713048002.pdf

Consejo Superior de Educación. (1994). La política educativa hacia el siglo XXI. Recuperado de: http://www.oei.es/quipu/costarica/politicaeducativasigloXXI.pdf

Consejo Nacional de Rectores (CONARE). (2011). III Informe del Estado de la Educación. (Programa Estado de la Nación en Desarrollo Humano Sostenible). Recuperado de http://www. estadonacion.or.cr/index.php/biblioteca-virtual/costa-rica/educacion 
Daft, R. y Marvic, D. (2006). Introducción a la administración. Argentina: Thompson.

Garbanzo, G. y Orozco, V. H. (2007). Desafíos del sistema educativo costarricense: un nuevo paradigma de la administración de la educación. Revista Educación, 31(2), 95-110. Recuperado de http://www.latindex.ucr.ac.cr/educacion-31-2/06-GARBANZO.pdf

Hax, A. y Majluf, N. (2004). Estrategias para el liderazgo competitivo. De la visión a los resultados. Buenos Aires: Granica.

La Gaceta. (21 de setiembre de 2009). Leyes y reglamentos docentes (Modificación del Decreto Ejecutivo № 35355-MEP. Reglamento de Evaluación de los Aprendizajes). Recuperado de http://www.apse.or.cr/webapse/legdoc/leg03.htm

Ministerio de Educación Pública de Costa Rica (MEP). (2011). Manual de procedimientos de control interno para centros educativos. Recuperado de http://circuito2pz.blogspot.com/2011/03/ manual-de-procedimientos-de-control.html

Ministerio de Educación Pública de Costa Rica [MEP]. (2011). Consideraciones respecto a las normas reguladoras de la promoción y repitencia dentro del sistema educativo público costarricense. Recuperado de http://www.mep.go.cr/downloads/Despacho/NORMAS\%20 DE\%20REPITENCIA.pdf

Rodríguez, J. L. y Gallegos, S. (1992). Lenguaje y rendimiento académico: un estudio en educación secundaria. España: Universidad de Salamanca.

Ugalde, N. (2011). Calidad en la gestión: administración por procesos, costeo por actividades y el cuadro de mando integral. Revista Ciencias Económicas, 29(2), 429-447. Recuperado de http://www.latindex.ucr.ac.cr/econ-29-2/econ-29-2-17.pdf

Vargas, I. (enero-abril, 2008). Análisis de cinco desafíos en el ejercicio de la administración educativa. Revista Actualidades Investigativas en Educación, 8(1), 1-15. Recuperado de http:// redalyc.uaemex.mx/pdf/447/44780117.pdf

Veciana, J. M. (1998). Función directiva. Barcelona: Universidad Autónoma de Barcelona.

Venegas, P. (2011). Gestión de la educación basada en competencias: Elementos para su interpretación en el contexto de la administración de la educación. Revista Gestión de la Educación, 1(1), 1-27. Recuperado de http://www.latindex.ucr.ac.cr/gestion-1-1/ gestion-1-1-03.pdf

Zayas, F.y Rodríguez, A. T. (enero-abril, 2010). Educación y educación escolar. Revista Actualidades investigativas en educación, 10(1), 2010, 1-21. Recuperado de http://revista.inie.ucr.ac.cr/ uploads/tx magazine/escolar.pdf 